\title{
Multiple aneurysms of the anterior communicating artery
}

\author{
KELLY K. KOELLER, LCDR, MC, USNR \\ ROBIN E. OSBORN, LCDR, MC, USNR
}

Multiple aneurysms of the anterior communicating artery are rare. The authors describe a patient who was admitted with a sudden, severe headache with radiation to the neck. Angiograms demonstrated filling of both anterior cerebral arteries and bilateral aneurysms arising from the origin of the anterior communicating artery. Both aneurysms were surgically verified and clipped.

(Key words: Anterior communicating artery, angiography, cerebral aneurysm)

Multiple cerebral aneurysms occur in approximately $20 \%$ of patients with cerebral aneurysms, but multiple aneurysms of the same artery are much less common. ${ }^{1,2}$ We present the angiographic findings in a rare case of multiple aneurysms of the anterior communicating artery.

\section{Report of case}

A 46-year-old right-handed woman suddenly had a severe headache with radiation to the neck. She did not lose consciousness but vomited once. On initial examination, her blood pressure was 170 $110 \mathrm{~mm} \mathrm{Hg}$. She was sleepy but arousable and had slight nuchal rigidity. The patient was neurologi-

From the Departments of Radiology and Clinical Investigation, Naval Hospital, San Diego, California; Department of Radiology, University of California, San Diego, California. Currently, Dr Osborn is at the Department of Radiology, Mercy Medical Center, Springfield, Ohio, and Dr Koeller is at the Department of Radiology, Neuroradiology Section, University of California-San Francisco Medical Center, San Francisco, Calif.

The work reported herein was performed under the Navy Clinical Investigation Program, case report no. 8416-1968-82. The views expressed in this article are those of the authors and do not reflect the official policy or position of the Department of the Navy, Department of Defense, nor the US Government.

Reprint requests to Robin E. Osborn, DO, Mercy Medical Center, Department of Radiology, 1343 N Fountain Rd, Springfield, OH 45501. cally intact and oriented with the exception of place. A computed tomographic scan of the head, performed shortly after the onset of symptoms, demonstrated blood in the anterior interhemispheric and sylvian fissures bilaterally.

Left internal carotid angiography demonstrated filling of both anterior cerebral arteries and aneurysms arising from the origin of the anterior communicating artery $(\mathrm{ACoA})$ bilaterally. A $5 \times 4$ $\mathrm{mm}$ aneurysm from the ACoA origin on the left and a second aneurysm, measuring $5 \times 3 \mathrm{~mm}$, on the right, were seen (Figure). An angiogram of the right internal carotid artery revealed a hypoplastic horizontal (A1) segment of the right anterior cerebral artery. Findings of left vertebral angiography were normal.

Four days after admission, the patient had rightsided weakness with increased lethargy. She gradually improved until 16 days after admission. She then had a recurrent intracranial hemorrhage. The aneurysms were verified surgically as aneurysms of bilateral ACoA origin and were successfully clipped.

\section{Discussion}

The ACoAs account for $23 \%$ to $35 \%$ of all cerebral aneurysms and $90 \%$ of all anterior cerebral artery aneurysms. ${ }^{3,4}$ Previous studies have demonstrated that in patients with aneurysms $17 \%$ had multiple aneurysms and $2.8 \%$ had multiple aneurysms of the same artery, with involvement of the internal carotid artery being the most common. ${ }^{5}$

Multiple aneurysms of the same artery have previously been noted to have an association with familial aneurysms and cerebral arteriovenous malformations. ${ }^{1}$ Anomalies of the horizontal segment of the anterior cerebral artery, including hypoplasia of the A1 segment, have been noted to show a slightly increased incidence of $\mathrm{ACoA}$ aneurysms. ${ }^{6}$

In our literature review, only eight cases of bilateral $\mathrm{ACoA}$ aneurysms were found. In one report, only one aneurysm was apparent angi- 

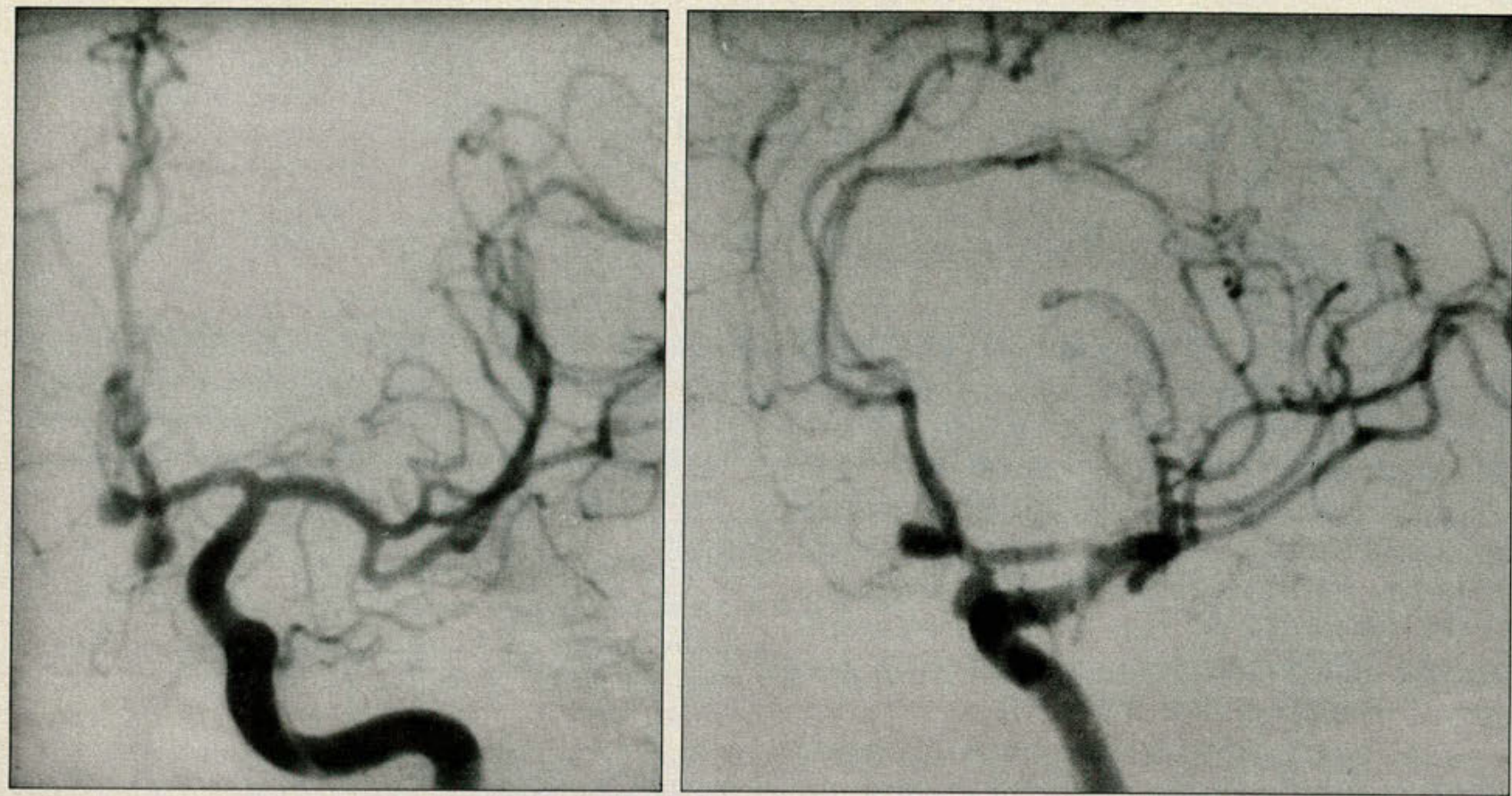

Figure. Left internal carotid angiogram. Posterior-anterior (Left) and oblique (Right) views demonstrate two aneurysms arising from the region of the anterior communicating artery. They were surgically proved to be aneurysms of bilateral anterior-communicating artery origin.

ographically while the second aneurysm was identified at surgery. ${ }^{5}$ The remaining seven cases were identified either angiographically or at autopsy. ${ }^{2}$ They were contained within a study of 2,951 patients with proved aneurysms. The method of detection for each individual case was not specifically stated, and no such cases were illustrated angiographically. To our knowledge, the case reported here is the first case of bilateral ACoA aneurysms demonstrated angiographically to be described in the medical literature.

Typically, in patients with multiple aneurysms, the larger aneurysm is the most likely to rupture. ${ }^{4,5}$ The aneurysms in our patient were nearly the same size, and no determination was made as to which aneurysm had bled. There were no clues (that is, spasm, mass ef- fect, or irregularity of the aneurysm) to identify which aneurysm had ruptured.

1. Garza-Mercado R, Rangel RA, Garza-Vazquez JF: Coexistence of bilateral aneurysms of the internal carotid arteries and an arteriovenous malformation of the left frontal lobe. Surg Neurol 1984;21:267-271.

2. Locksley HB: Report on the cooperative study of intracranial aneurysms and subarachnoid hemorrhage: Section V, Part I: Natural history of subarachnoid hemorrhage, intracranial aneurysms and arteriovenous malformations. J Neurosurg 1966;25:219-239.

3. Handa J, Nakasu Y, Matsuda M, et al: Aneurysms of the proximal anterior cerebral artery. Surg Neurol 1984;22:486-490.

4. Osborn AG: Circle of Willis, in Osborn AG: Introduction to Cerebral Angiography. Philadelphia, Harper \& Row, 1980, pp 145-165.

5. Kojima T, Waga S: More than one aneurysm on the same artery. Surg Neurol 1984;22:403-408.

6. Osborn AG: The anterior cerebral artery, in Osborn AG: Introduction to Cerebral Angiography. Philadelphia, Harper \& Row, 1980, pp 185-237. 
When diet and exercise alone are not enough...

\section{A MILD START IN MILD HYPERTENSION}
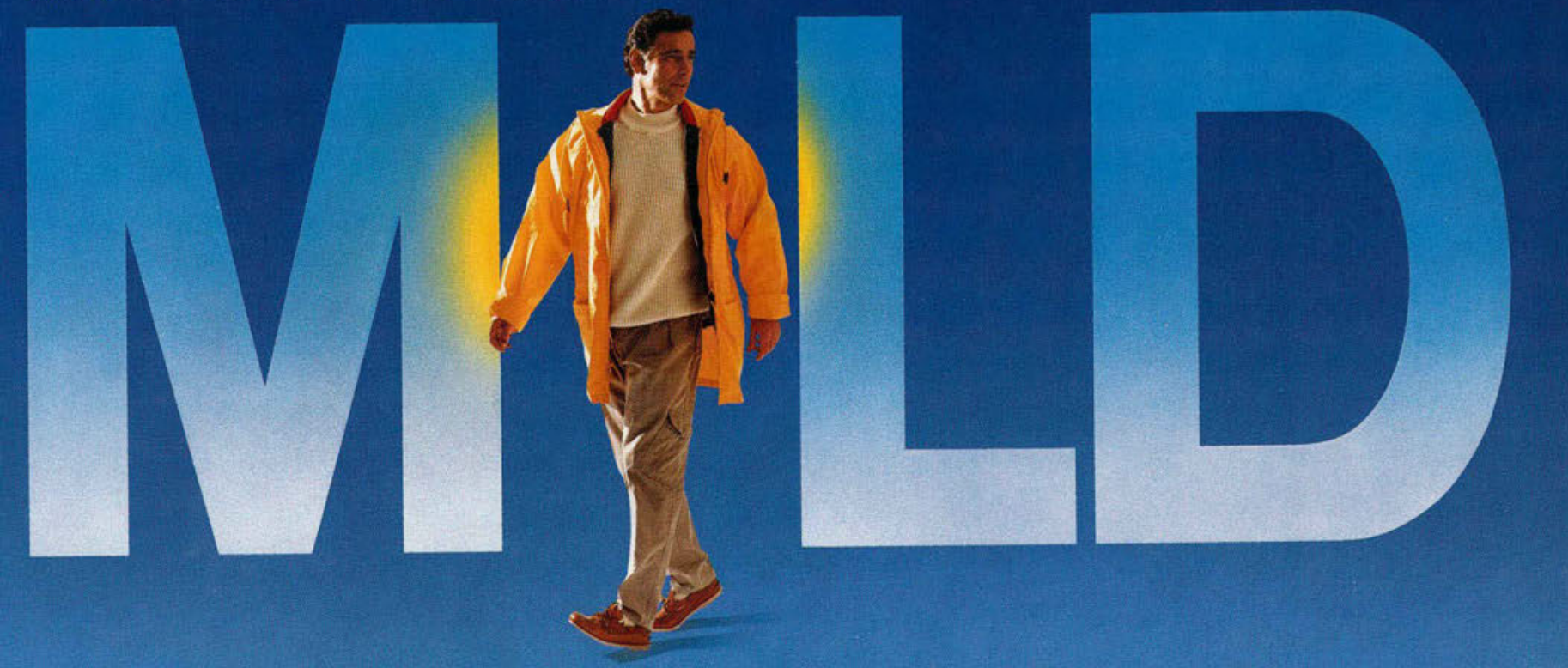

- Single-agent efficacy

- Low incidence of side effects

- Once-daily $180 \mathrm{mg}$ - the recommended starting dose for Calan SR

- Scored caplet provides easy titration

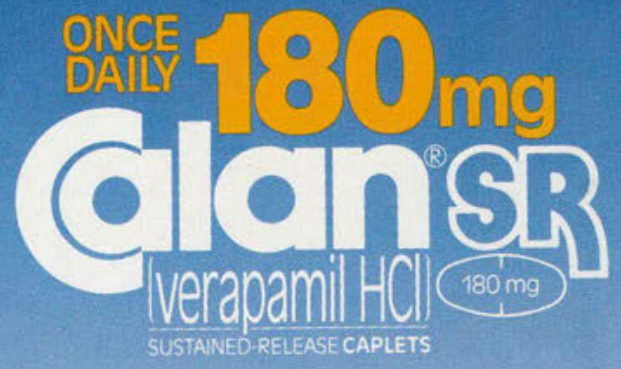

References:

1. Data on file, C.D. Searle \& Co. 2. Midtbø KA. Effects of long-term verapamil therapy on serum lipids and other metabolic parameters. Am J Cardiol. 1990:66:131-151. 3. Krone W, Nàgele H. Effects of antihypertensives on plasma lipids and lipoprotein metabolism. Am Heart J.1988:116:1729:1734. 4. Cruickshank JK. Anderson NMCF. Wadsworth J, et al. Treating hypertensive diabetics: a comparison of verapamil and metoprolol in black and white patients J CardiovasC Pharmacol 1987;10(suppl 10): S85-S86. 5. Schmieder RE, Messerlt $\mathrm{FH}$, Garavaglia GE, et al. Cardiovascular effects of verapamil in patients with essential hypertension. Circulation. 1987;75:1030-1036.

\section{BRIEF SUMMARY}

Contraindications: Severe LV dysfunction (see Warnings), hypotension (systolic pressure $<90 \mathrm{~mm} \mathrm{Hg}$ ) or cardiogenic shock, sick sinus syndrome (if no pacemaker is present), 2ndor 3rd-degree AV block (if no pacemaker is present), atrial flutter/fibrillation with an accessory bypass tract (eg. WPW or LGL syndromes), hypersensitivity to verapamil.

Warnings: Verapamil should be avoided in patients with severe LV dysfunction (eg, ejection fraction $<30 \%$ ) or moderate to severe symptoms of cardiac failure and in patients with any degree of ventricular dysfunction if they are receiving a beta-blocker. Control milder heart failure with optimum digitalization and/or diuretics before Calan SR is used. Verapamil may occasionally produce hypotension. Elevations of liver enzymes have been reported. Several occasionally produce hypotension. Elevations of liver enzymes have been reported. Several
cases have been demonstrated to be produced by verapamil. Periodic monitoring of liver function in patients on verapamil is prudent. Some patients with paroxysmal and/or chronic atrial flutter/fibrillation and an accessory AV pathway (eg. WPW or LGL syndromes) have developed an increased antegrade conduction across the accessory pathway bypassing the AV node, producing a very rapid ventricular response or ventricular fibrillation after receiving I.V. verapamil (or digitalis). Because of this risk, oral verapamil is contraindicated in such patients. AV block may occur (2nd-and 3rd-degree, 0.8\%). Development of marked 1stdegree block or progression to 2 nd- or 3 rd-degree block requires reduction in dosage or. rarely discontinuation and institution of appropriate therapy. Sinus bradycardia, 2 nd-degree AV block, sinus arrest, pulmonary edema and/or severe hypotension were seen in some critically ill patients with hypertrophic cardiomyopathy who were treated with verapamil.

Precautions: Verapamil should be given cautiously to patients with impaired hepatic function (in severe dysfunction use about $30 \%$ of the normal dose) or impaired renal function, and patients should be monitored for abnormal prolongation of the PR interval or other signs of overdosage. Verapamil may decrease neuromuscular transmission in patients with Duchenne's muscular dystrophy and may prolong recovery from the neuromuscular blocking agent vecuronium It may be necessary to decrease verapamil dosage in patients with attenuated neuromuscular transmission. Combined therapy with beta-adrenergic blockers and verapamil may result in additive negative effects on heart rate, atrioventricular conduction and/or cardiac contractility; there have been reports of excessive bradycardia and AV block, including complete heart block. The risks of such combined therapy may outweigh the benefits. The combination should be used only with caution and close monitoring. Decreased metoprolol and propranolo clearance may occur when either drug is administered concomitantly with verapamil. A variable effect has been seen with combined use of atenolol. Chronic verapamil treatment can increase serum digoxin levels by $50 \%$ to $75 \%$ during the first week of therapy, which can result in digitalis toxicity In patients with hepatic cirrhosis, verapamil may reduce total body clearance given, and the patient carefully monitored. Verapamil will usually have an additive effect in

\section{Calan SR 180-mg caplets are scored for easy titration.}

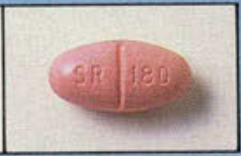

patients receiving blood-pressure-lowering agents. Disopyramide should not be given within 48 hours before or 24 hours after verapamil administration. Concomitant use of flecainide and verapamil may have additive effects on myocardial contractility, AV conduction, and repolarization. Combined verapamil and quinidine therapy in patients with hypertrophic cardiomyopathy should be avoided, since significant hypotension may result. Concomitant use of lithium and verapamil may result in a lowering of serum lithium levels or increased sensitivity to lithium. Patients receiving both drugs must be monitored carefully. Verapamil may increase carbamazepine concentrations during combined use. Rifampin may reduce verapamil bioavailability Phenobarbital may increase verapamil clearance. Verapamil may increase serum levels of cyclosporin. Verapamil may inhibit the clearance and increase the plasma levels of theophylline. Concomitant use of inhalation anesthetics and calcium antagonists needs careful titration to avoid excessive cardiovascular depression. Verapamil may potentiate the activity of neuromuscular blocking agents (curare-like and depolarizing); dosage reduction may be required. Adequate animal carcinogenicity studies have not been performed. One study in rats did not suggest a tumorigenic potential, and verapamil was not mutagenic in the Ames test. Pregnancy Category C. There are no adequate and well-controlled studies in pregnant women. This drug should be used during pregnancy, labor, and delivery only if clearly needed. Verapamil is excreted in breast milk; therefore, nursing should be discontinued during verapamil use.

Adverse Reactions: Constipation $(7.3 \%)$, dizziness $(3.3 \%)$, nausea $(2.7 \%)$, hypotension $(2.5 \%)$, headache $(2.2 \%)$, edema $(1.9 \%)$, CHF, pulmonary edema $(1.8 \%)$, fatigue $(1.7 \%)$, dyspnea $(1.4 \%)$, bradycardia: $H R<50 /$ min $(1.4 \%)$, AV block: total $1^{\circ}, 2^{\circ}, 3^{\circ}(1.2 \%), 2^{\circ}$ and $3^{\circ}(0.8 \%)$, rash $(1.2 \%)$, flushing $(0.6 \%)$, elevated liver enzymes, reversible non-obstructive paralytic ileus The following reactions, reported in $1.0 \%$ or less of patients, occurred under conditions where a causal relationship is uncertain: angina pectoris, atrioventricular dissociation, chest pain, claudication, myocardial infarction, palpitations, purpura (vasculitis), syncope, diarrhea, dry mouth, gastrointestinal distress, gingival hyperplasia, ecchymosis or bruising, cerebrovascula accident, confusion, equilibrium disorders, insomnia, muscle cramps, paresthesia, psychotic symptoms, shakiness, somnolence, arthralgia and rash, exanthema, hair loss, hyperkeratosis, macules, sweating, urticaria, Stevens-Johnson syndrome, erythema multiforme, blurred vision, gynecomastia, galactorrhea/hyperprolactinemia, increased urination, spotty menstruation impotence.

4/11/91 - P91CA6143V

Address medical inquiries to:

G.D. Searle \& Co.

Medical \& Scientific

Information Departm
4901 Searle Parkway

4901 Searle Parkw
Skokie, IL 60077

SEARLE

G.D. Searle \& Co

Box 5110, Chicago, IL 60680 
When diet and exercise alone are not enough...
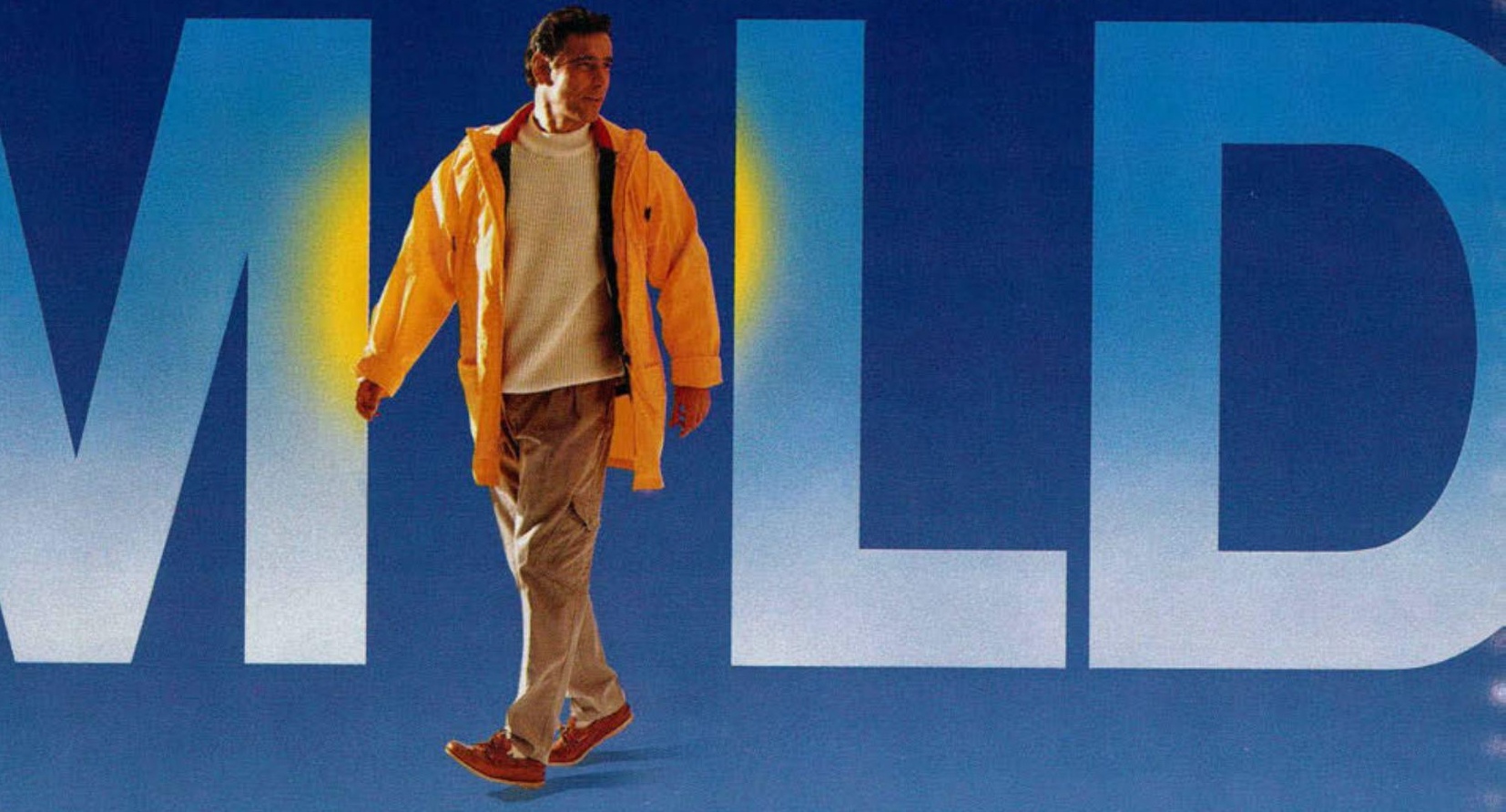

\title{
A MILD START IN MILD HYPERTENSION
}

\author{
- SINCLE-ACENT EFFICACY \\ - ONCE-DAILY STARTING DOSE \\ - EXCELLENT TOLERABILITY
}

- NO ADVERSE EFFECTS ON LIPID AND GLUCOSE

LEVELS $^{2 \cdot 4}$ OR ON RENAL FUNCTION

The recommended starting dose for Calan SR is $180 \mathrm{mg}$ once daily. Dose titration will be required in some patients to achieve blood pressure control.

A lower initial starting dosage of $120 \mathrm{mg} / \mathrm{day}$ mav be

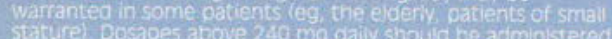
in oivided doses. Calan SR shoula be administered wath food tConstipation. which is easiy managed in most patients, is the

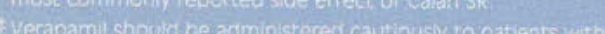
imnaired renal functing

Please see next page of this aovertisement for references and : briaf summary of preseribing information
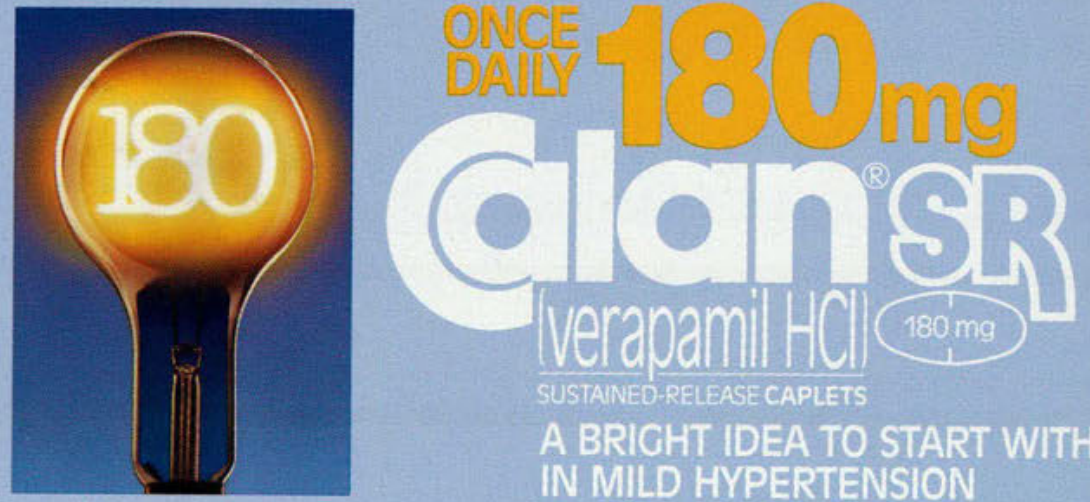

A BRICHT IDEA TO START WITH IN MILD HYPERTENSION 